\title{
Indication of linkage and genetic heterogeneity of asthma according to age at onset on chromosome $7 q$ in 107 French EGEA families
}

\author{
Marie-Hélène Dizier*,1, Céline Besse-Schmittler ${ }^{2}$, Michel Guilloud-Bataille ${ }^{1}$, \\ Hana Selinger-Leneman ${ }^{1}$, Francine Kauffmann ${ }^{3}$, Françoise Clerget-Darpoux ${ }^{1}$ and \\ Florence Demenais ${ }^{2}$
}

\author{
${ }^{1}$ INSERM U535, Kremlin-Bicêtre, Cedex, France; ${ }^{2}$ INSERM-EPI 00-06, Paris, France; ${ }^{3}$ INSERM U472, Villejuif, \\ France
}

It is generally believed that an early age at the onset of disease is associated with a stronger genetic component. Our aim here was to investigate both linkage and genetic heterogeneity of asthma, the latter corresponding to different genotype relative risks of a putative linked gene according to age at onset of asthma. This analysis was conducted in 107 French EGEA families with at least two asthmatic siblings, considering 157 markers that were part of our previous genome screen, using the TTS (the Triangle Test Statistic) which has been developed to detect both linkage and intra-sibpair genetic heterogeneity. This test has been applied to 38 asthmatic sib-pairs discordant for age at the onset of asthma. To confirm the existence of genetic heterogeneity, we also used the predivided sample test (PST) which compares the IBD (identity by descent) distribution of marker alleles between asthmatic sib-pairs concordant (67) and discordant (38) for the age at onset. The cutoff point used for the age at onset was 4 years, the median age at onset in our sample of asthmatic sibs. Linkage and genetic heterogeneity for a region located on chromosome $7 \mathrm{q}$ (at $109 \mathrm{cM}$ from pter) were indicated by both tests, TTS $(P=0.005, P>0.5$ after correction for multiple testing) and PST ( $P=0.0001,0.015$ after correction). These results suggest a genetic factor on $7 q$ involved in asthma with genotype relative risks differing according to age at onset of disease. European Journal of Human Genetics (2001) 9, 867-872.

Keywords: linkage; genetic heterogeneity; genotype risks; asthma; age at onset

\section{Introduction}

Asthma is a complex disorder that is likely to depend on numerous genetic and environmental factors. At the present time, several genome-wide searches and candidate gene studies have reported many regions potentially linked to asthma and related traits (see the review of Ober and Moffatt). ${ }^{1}$ Our genome wide search ${ }^{2}$ has led to detect seven

*Correspondence: Marie-Hélène Dizier, Unité INSERM U535, Bâtiment INSERM Gregory Pincus, 80 rue du Général Leclerc, 94276 Le Kremlin Bicêtre Cedex, France. Tel: 331495953 30: Fax: 331495953 31; E-mail: dizier@kb.inserm.fr

Received 13 July 2001; revised 6 September 2001; accepted 14 September 2001 regions, also found by previously published genome searches. ${ }^{3-6}$ Two of these regions, 1 p31 and $17 q 12-21$, were detected with potential linkage for asthma only and for asthma and skin tests respectively. The other regions were detected for related phenotypes, 11p13 and 11q13 for IgE levels, 12q24 and 13q31 for eosinophils and 19q13 for bronchial responsiveness.

However, in most previous studies, linkage analyses were conducted for asthma and/or related phenotypes separately, and did not take into account the phenotypical heterogeneity of asthma and its association with other traits. Asthma is indeed a heterogeneous disease which presents a large clincial spectrum. The pattern of asthma varies according to age and gender, ${ }^{7}$ it is heterogeneous regarding 
its association with atopy (intrinsic/extrinsic asthma) ${ }^{8}$ and exhibits various patterns regarding its severity. ${ }^{9}$ Besides potential differences in the management of the patients, the phenotypic heterogeneity is likely to be related to the interplay of various environmental and genetic etiological factors. Phenotypic heterogeneity may correspond to genetic heterogeneity.

It is generally believed that an early age at the onset of disease is associated with a stronger genetic component and accounting for the age at onset in linkage analysis may increase power to detect linkage. It has often been an important step in the identification of a number of disease genes, such as BRCA1 for breast cancer. ${ }^{10}$

Using the sample of 107 French families selected through two asthmatic siblings as a part of the EGEA study (Epidemiological study on the Genetics and Environment of Asthma), our aim here was to investigate both linkage and genetic heterogeneity of asthma, the latter corresponding to different relative genotype risks of a linked gene according to the age at onset of asthma. The Triangle Test Statistic (TTS) ${ }^{11}$ developed to detect both linkage and intra-sib-pair genetic heterogeneity, ie the sibs differing for a factor on which the relative genotype risks of the linked gene depend, was applied to asthmatic sib-pairs discordant for age at onset of asthma using a set of 157 markers that were part of our previous genome screen. To confirm the existence of genetic heterogeneity according to asthma age at onset, the predivided sample test (PST) was also used to compare the IBD (identity by descent) distribution of marker alleles between asthmatic sib-pairs concordant and discordant for age at onset.

\section{Material}

\section{Family data}

As part of the EGEA study, a sample of 119 families with at least two asthmatic siblings and at most one asthmatic parent was collected for the genome-wide search. Asthmatic siblings of these families met the following criteria: (i) current age above 7 years; (ii) born in France as well as his/her parents; (iii) a positive response to at least one of the two questions (1) Have you ever had attacks of breathlessness at rest with wheezing? (2) Have you ever had an asthma attack?), associated with either the presence of BHR (defined as a fall in baseline $\mathrm{FEV}_{1}$, the forced expiratory volume at $1 \mathrm{~s}, \geqslant 20 \%$ at $\leqslant 4 \mathrm{mg} / \mathrm{ml}$ methacholine) or $\geqslant 15 \%$ increase of baseline $\mathrm{FEV}_{1}$ after bronchodilator use, hospitalisation for asthma in life or asthma therapy. ${ }^{12,13}$ After excluding families with insufficient DNA available or showing non-mendelian transmission, the analysed sample consisted of 107 families with a total of 493 individuals. In the 279 genotyped siblings of the 107 families, $59 \%$ of siblings were males and the mean age was $14.5 \pm 7.6(\mathrm{SD})$ years.

The protocol was approved by ethical committee and subjects signed informed consent forms.

\section{Clinical evaluation}

Information on respiratory and allergic symptoms, medical history and environmental factors was recorded on a questionnaire. The following tests were performed:

(1) skin prick tests to 11 allergens, ie cat, Dermatophagoides pteronyssinus, Cladosporium herbarum, Alternaria tenuis, timothy grass, olive, birch, Parieteria judaica, ragweed, Aspergillus, Blatella Germanica.

(2) total immunoglobulin E concentration measured by radioimmunoassay (Phadebas PRIST technique; Pharmacia diagnostics, AB, France) in one central laboratory. ${ }^{14}$

(3) total and differential white blood cell counts were assessed by manual or automating reading according to the usual method in each center.

(4) basal spirometry was conducted according to the ECRHS protocol ${ }^{15}$ with slight modifications and including methacholine bronchial challenge test.

\section{Genotypes}

Genomic DNA extraction as well as methods for genotype determination and checking were described elsewhere. ${ }^{2}$ We used for our genome scan a two-stage analysis strategy with an initial screen in a first set of 46 families including 254 autosomal microsatellite markers with an average spacing of $13 \mathrm{cM}$ and an average heterozygosity over all loci of 0.78 . Markers tested in the second set of 61 families for replication, ie belonging to all regions detected in the first set of families and to regions reported by published genome screens, represented $70 \%$ of markers (157 markers) of the initial screen. To obtain sufficient sizes of asthmatic sib-pairs discordant and concordant for age at onset, we considered the whole sample of 107 families and thus the 157 markers tested in this sample.

\section{Phenotypes analysed}

For linkage analysis, asthma was defined as indicated above for the selection of the 107 families. In the 279 genotyped siblings of the 107 families, $83.7 \%$ were asthmatic. In this sample, the age at first asthma attack ranged between 0 and 24 years, with a mean of $5.3 \pm 5.13$ (SD) years and a median of 4 years. We used the age of 4 years as the cutoff-point to separate the very early ( $\leqslant 4$ years) and less early age at onset ( $>4$ years) of asthmatic sibs. There were 115 independant asthmatic sib-pairs (in a sibship with $n$ affected sibs, $n$ - 1 pairs were considered), including 48 pairs with both sibs having an age at onset $\leqslant 4$ years, 29 pairs with both sibs having an age at onset $>4$ years and 38 pairs discordant for age at onset.

\section{Analysis}

Linkage analysis by IBD sharing The principle of sib-pair linkage analysis is to assess the sharing of marker alleles identical by descent (IBD) among affected sib-pairs and to conclude in favour of linkage if the observed IBD distribution differs from that expected under the null hypothesis of independence of segregation of the disease and markers. This 
comparison can be made with the MLS statistic. ${ }^{16}$ The MLS is based on a maximum likelihood method which estimates the proportions of sib-pairs IBD=2, 1 or 0 (respectively z2, z1 and $\mathrm{z0})$, given the observed marker genotypes. It has been shown ${ }^{17}$ that, if a susceptibility gene is assumed to be linked to the marker, the proportions $\mathrm{z} 2, \mathrm{z} 1$ and $\mathrm{z} 0$ expected among affected sib-pairs are constrained by: $2 z 0 \leqslant z 1 \leqslant 0.5$, referred as the triangle constraints. ${ }^{18}$ However, Dizier et al ${ }^{11}$ showed that if the phenotype of each member of a sib-pair is determined by different genetic models, ie different relative genotype risks (the relative genotype risks being the ratios of the genotype-specific penetrances to the penetrance of homozygotes for the disease allele) for the phenotype of each sib, the triangle constraints may not be valid. This situation can occur when the two sibs differ for a factor that modifies the genotype relative risks for the disease. This factor may be specific to the trait itself, eg severe $v s$ mild form of the disease, or the presence/absence of an associated trait or an environmental factor. We also showed that under some models of heterogeneity, departure from the triangle constraints may be large and using the MLS test with the triangle constraints may substantially decrease the power to detect linkage. ${ }^{11}$

TTS method The Triangle Test Statistic, ${ }^{11}$ called TTS, has been introduced to test whether the triangle constraints in marker IBD distribution among affected sib-pairs are fulfilled. This test is defined as:

$$
\text { TTS }=\log _{10}\left(\mathrm{~L}\left(\mathrm{Z}_{\mathrm{u}}\right) / \mathrm{L}\left(\mathrm{Z}_{\mathrm{c}}\right)\right)
$$

where $L\left(Z_{c}\right)$ is the likelihood of the IBD vector estimated with the triangle constraints, and $\mathrm{L}\left(\mathrm{Z}_{\mathrm{u}}\right)$ is the likelihood without these constraints. This ratio is equivalent to the difference of the MLS scores obtained with and without constraints. The null hypothesis HO is composite: absence of linkage or linkage with genetic homogeneity (ie relative penetrances are the same for the phenotypes of both members of the sibpair). Under H0, the TTS score is expected to be small. Rejection of HO means linkage with genetic heterogeneity within sib-pairs, ie the sibs' phenotypes differ for a factor on which the relative penetrances depend.

As Holmans ${ }^{18}$ did for the MLS distribution, we used the method of Self and Liang ${ }^{19}$ to calculate the asymptotic

Table 1 Tresholds of the Triangle Test Statistics (TTS) for various critical values $(\alpha)$ in the case of a single test using a marker with five alleles and typed parents

\begin{tabular}{ll}
\hline$\alpha$ & $T T S$ \\
\hline 0.05 & 1.06 \\
0.01 & 1.73 \\
0.001 & 2.72 \\
0.0001 & 3.71 \\
0.00001 & 4.70 \\
\hline
\end{tabular}

distribution of 2Ln10TTS (ie $2 \mathrm{Ln}\left(\mathrm{L}\left(\mathrm{Z}_{\mathrm{u}}\right) / \mathrm{L}\left(\mathrm{Z}_{\mathrm{c}}\right)\right)$ ) as a mixture of $\chi_{1 \text { df }}^{2}$ and $\chi_{2 \text { df }}^{2}$. Table 1 shows the thresholds $(\mathrm{K})$ of the Triangle Test Statistic corresponding to various critical values $(\alpha)$, which were calculated using the following formula $: \alpha=1$ / $2 \operatorname{Pr}\left(\chi^{2}{ }_{1 \mathrm{df}}>\mathrm{K}\right)+(\pi-\theta) / 2 \operatorname{Pr}\left(\chi^{2}{ }_{2 \mathrm{df}}>\mathrm{K}\right)$ with $\theta$ being the probability of being in the triangle and $\pi$ the usual pi value.

Note that we checked that critical values for given thresholds estimated from the asymptotical distribution as described just above were very close to the empirical $P$ values calculated by simulation studies for a small sample size of 38 discordant sib-pairs.

The predivided sample test (PST) Let us consider two subphenotypes, A and $\mathrm{A}^{\prime}$, which represent the affected status with either presence or absence of a given factor (here asthmatic with age at onset $\leqslant 4$ years and asthmatic with age at onset $>4$ years). Under the null hypothesis of genetic homogeneity (ie presence of the factor does not modify the relative penetrances), the vectors $Z_{\mathrm{u}}$ estimated, without the triangle constraints, among pairs concordant for the phenotype (both sibs being $\mathrm{A}$ or $\mathrm{A}^{\prime}$ ) and among discordant pairs (one sib being $A$, the other $A^{\prime}$ ) are expected to be equal. Therefore the null hypothesis can be tested by $-2\left[\operatorname{Ln}\left(\mathrm{L}\left(\mathrm{z}_{\mathrm{u}} 1\right) \cdot \mathrm{L}\left(\mathrm{z}_{\mathrm{u}} 2\right) / \mathrm{L}\left(\mathrm{z}_{\mathrm{u}}\right)\right)\right]$ which follows a $\chi^{2}$ distribution with $2 \mathrm{df}^{20}{ }^{20}$ where $\mathrm{L}\left(\mathrm{z}_{\mathrm{u}} 1\right), \mathrm{L}\left(\mathrm{z}_{\mathrm{u}} 2\right)$ and $\mathrm{L}\left(\mathrm{z}_{\mathrm{u}}\right)$ are the likelihoods of the parameter vector $Z_{u}$ estimated in the concordant sibpairs, discordant pairs and the whole sample respectively.

Strategy of Analysis We use here a different application of the TTS from the one initially introduced by Dizier et al. ${ }^{11}$ In our previous paper, we proposed to test linkage using the MLS without constraints followed by the TTS on the whole sample of sib-pairs to search for genetic heterogeneity. If we want to investigate genetic heterogeneity according to a specific factor of special interest, we can directly apply the TTS to discordant pairs for this factor in order to improve the power to detect genetic heterogeneity. Indeed the highest the proportion of discordant pairs for this factor, the largest the departure from the triangle constraints is observed. ${ }^{11}$ Since age at onset of asthma represents phenotypic heterogeneity for asthma, we applied here the TTS directly to discordant pairs for age at onset. Rejection of the triangle constraints by the TTS will suggest a linked genetic factor with different relative genotype risks in the two sub-phenotypes (asthmatics with age at onset $\leqslant 4$ or $>4$ years). To confirm potential genetic heterogeneity detected by TTS (markers with TTS value $>1.7$, threshold for a type 1 error of $1 \%$ ), we applied the PST among asthmatic pairs concordant and discordant for age at onset.

These tests were applied using all possible independent pairs in sibships (ie $(n-1)$ pairs per sibships with $n$ affected sibs) and a multipoint approach to estimate the IBD sharing, taking into account the information provided by the entire set of chromosome markers. For the TTS, the Bonferroni correction was used to account for the number of markers 
tested (157). Note that this correction ignores linkage between markers and for this reason is conservative, overestimating the type 1 error. Although the PST was applied only to markers detected by the TTS, we also used a Bonferroni correction for the total number of markers because of the non-independence between the two tests.

\section{Results}

\section{TTS and PST}

Results of the TTS and PST are given in Table 2. The TTS applied to 38 asthmatic pairs discordant for age at onset showed indication of linkage and genetic heterogeneity for one region located on chromosome $7 q$, with TTS values $>1.7$ for two markers D7S524 and D7S527, distant from $10.2 \mathrm{cM}$. The highest score is obtained at D7S527 locus, with a TTS value of 2.5 ( $P=0.005, P>0.5$ after correction). The IBD distribution among discordant affected sib-pairs at this marker (see Table 3) showed a large departure from the triangle constraints; in particular, the constraint $\mathrm{z} 1 \leqslant 0.5$ is strongly violated with an observed proportion equal to 0.81 . As the two chromosome 7 markers belong to the same region, we applied the PST only to the marker with the highest TTS score (ie D7S527). Genetic heterogeneity according to age at asthma onset was confirmed by the PST $(P=0.0001, P=0.015$ after correction) when comparing the estimated $Z$ vectors among asthmatic pairs discordant and concordant for age at onset. In concordant pairs, the $\mathrm{Z}$ vector was indeed very different from the one estimated in discordant pairs (see Table 3).

\section{Discussion}

Accounting for age at onset of asthma in our 107 EGEA families with at least two asthmatic siblings led us to detect a region on chromosome $7 \mathrm{q}$ potentially linked to asthma with genetic heterogeneity according to age at onset of asthma by using the Triangle Test Statistic. This suggests a linked genetic factor involved in asthma but with different relative genotype risks according to age at onset whether it is $\leqslant$ or $>4$ years. Note that this region was not detected in our previous genome scan, by linkage analysis of asthma or the main related traits (IgE, positive skin test response to at least one allergen, bronchial responsiveness, eosinophil count). However, we conducted a two-stage analysis strategy for our

Table 2 Results of the TTS applied to 157 markers and the PST applied to markers with TTS $>1.7$ in the sample of 38 asthmatic sib-pairs discordant for age at onset

\begin{tabular}{lllll}
\hline Markers & TTS & P value* & PST $\left(\chi^{2}{ }_{2 d f}\right)$ & P value $^{*}$ \\
\hline D7S524 & 2.4 & $0.006(>0.5)$ & & \\
D7S527 & 2.5 & $0.005(>0.5)$ & 18.4 & $0.0001(0.015)$ \\
\hline
\end{tabular}

* In parentheses, $P$ values after the Bonferroni correction. genome scan and in our first set of data (46 families), a potential linkage was detected between D7S527 (the marker showing the maximum TTS score) and asthma $(P=0.007$, not corrected $P$ value). This result was not replicated in the second data set (61 families) and led in the whole sample to a $\mathrm{p}$ value of 0.35 . A non replication could also be due to heterogeneity between samples, however we checked that clinical characteristics of the two sub-samples did not differ significantly. ${ }^{13}$ All these results might be explained by the fact that ignoring genetic heterogeneity, by classical linkage analysis, may reduce greatly the power to detect linkage. Under some models of heterogeneity and when the proportion of discordant sib-pairs is substantial $(>0.5)$, the sample size necessary to detect linkage by the MLS with constraints may be infinite. In such situations, the departure from the constraints is large and the use of constraints to estimate the IBD vector leads to the distribution expected under no linkage $(1 / 4,1 / 2,1 / 4) .{ }^{11}$ It is thus much more powerful to use the MLS without constraints or the TTS to detect linkage and/or genetic heterogeneity. Another interesting illustration of the TTS was provided by an analysis of simulated data including sib-pairs affected by a disease having two forms (severe or mild), as part of an international Genetic Analysis Worshop (GAW 11). ${ }^{21}$ The TTS led to the conclusion that a genetic factor linked to one of the markers studied had a different involvement in the severe and mild forms of the disease.

Different ways of stratifying the data, eg according to the phenotypic status of the index only (affected with or without a phenotypic factor) or according to the phenotypic status of the two sibs of a pair, using both concordant and discordant sib-pairs or using discordant sib-pairs only (as presented here), can be used to detect genetic heterogeneity by linkage analysis. In the present study, the departure from the triangle constraints in the IBD distribution of discordant pairs or the difference in IBD distribution between discordant and concordant pairs permits to demonstrate the presence of heterogeneity. However power of different approaches of stratification is likely to depend on the underlying model of heterogeneity.

Most of the published genomewide searches of asthma $^{3-6,22-26}$ did not report the $7 q$ region, except a recent genome search ${ }^{27}$ in families from Netherlands which reported linkage between this region and total IgE levels, the distance between the marker which reached the maximum lod-score in this study and D7S527 being only $0.5 \mathrm{cM}$. The gene detected on $7 q$ could be a modifier gene, specifically involved in age at asthma onset. Another possible model might be a susceptibility gene linked to asthma with an early age at onset in subjects homozygous for the 'disease' allele and a less early age at onset in heterozygotes. We showed that such a model would lead to a substantial departure from the triangle constraints. ${ }^{11}$ In constrast, the two regions, $1 \mathrm{p}$ and $17 \mathrm{q}$, which were detected as being potentially linked to asthma by our previous genome scan, 
Table 3 IBD distributions at the marker D75527 among asthmatic sib-pairs concordant and discordant for the age at onset $(\leqslant />4$ years)

\begin{tabular}{llll}
\hline Sib-pair & $Z 0$ & $Z 1$ & $Z 2$ \\
\hline Concordant & 0.27 & 0.34 & 0.39 \\
Discordant & 0.10 & 0.81 & 0.09 \\
\hline
\end{tabular}

were not detected here by the TTS for genetic heterogeneity according to age at onset of asthma.

The median of age at onset of 4 years in our sample of asthmatic siblings was chosen here as a cutoff point to search for genetic heterogeneity according to age at onset of asthma. It should be noticed that the alveolar growth runs about 36 weeks to term and continues for at least 3 years of postnatal life, during which alveoli multiply greatly in number. Since the age of the end of alveolar growth is not precisely determined and may vary from one individual to another, 4 years might correspond to a fairly good approximation of this age.

The next step would be to try to replicate our results in other data sets and then to search for associations of asthma with candidates genes on 7q. The cystic fibrosis transmenbrane conductance regulator gene (CFTR) is located $16 \mathrm{cM}$ apart from the region detected in the present study. Association studies have suggested that $\Delta \mathrm{F} 508$ heterozygosity $^{28}$ in the CFTR gene and other variants ${ }^{29}$ could be risk factors for asthma but such association was not confirmed in the EGEA study. ${ }^{30}$ Other candidate genes need to be further investigated.

\section{EGEA co-operative group}

Respiratory epidemiology I Annesi-Maesano, F Kauffmann (co-ordinator), MP Oryszczyn (INSERM U472 (ex-U169), Villejuif); F Neukirch, M Korobaeff (INSERM U408, Paris).

Genetics MH Dizier (INSERM U535 (ex-U155), Paris), J Feingold (INSERM U393 (ex-U155), Paris), F Demenais (INSERM EPI 00-06 (ex-U358), Paris), M Lathrop (Centre National de Génotypage, Evry, France).

Clinical centres Grenoble : I Pin, C Pison; Lyon : D Ecochard (deceased), F Gormand, Y Pacheco ; Marseille : D Charpin, D Vervloet ; Montpellier : J Bousquet; Paris Cochin : A Lockhart, R Matran (now in Lille); Paris Necker : E Paty, P Scheinmann ; Paris-Trousseau : A Grimfeld.

Data management J Hochez (INSERM U155), N Le Moual (INSERM U472).

\section{Acknowledgments}

This work was supported by convention INSERM-MSD, INSERM networks of clinical research (489012) and public health research
(493009) and INSERM grant (IDS-4D006D). Kits for IgE and Phadiatop determinations were kindly provided by Pharmacia.

\section{References}

1 Ober C, Moffatt M: Contributing factors to the pathobiology : the genetics of asthma. In: Wenzel S (ed) The pathobiology of asthma. WB Saunders, Philadelphia, 2000, vol 21, p 245-261.

2 Dizier MH, Besse-Schmittler C, Guilloud-Bataille $\mathrm{M}$ et al: Genome screen for asthma and related phenotypes in the French EGEA study. Am J Respir Crit Care Med 2000; 162: 1812 - 1818.

3 Daniels SE, Bhattacharrya S, James A et al: WOC: A genome-wide search for quantitative trait loci underlying asthma. Nature 1996; 383: 247-250.

4 The Collaborative Study on the Genetics of Asthma (CSGA): A genome-wide search for asthma susceptibility loci in ethnically diverse populations. Nature Genetics 1997; 15: 389-392.

5 Ober C, Cox NJ, Abney M et al: Genome-wide search for asthma susceptibility loci in a founder population. Hum Mol Genet 1998; 7: $1393-1398$.

6 Wjst M, Fisher G, Immervoll T et al: A genome-wide search for linkage to asthma. Genomics 1999; 58: $1-8$.

7 Kauffmann F, Becklake MR: Sex and gender. In : Respiratory epidemiology in Europe. Annesi-Maesano I, Viegi G, Gulsvik A eds. Eur Respir Mon 2000; 14: 288-304.

8 Rackeman FM: A working classification of asthma. Am J Med 1947; 33: 601-606.

9 Wenzel, S.E: Factors determining the severity of asthma. Clin Exp Allergy 1998; 28 (suppl 5): 119-125.

10 Hall JM, Lee MK, Newman B et al: Linkage of early-onset familial breast cancer to chromosome 17q21. Science 1990; 250: $1684-$ 1689.

11 Dizier MH, Quesneville H, Prum B, Selinger-Leneman H, Clerget-Darpoux F: The triangle test statistic (TTS) : a test of genetic homogeneity using the departure from the triangle constraints in IBD distribution among affected sib-pairs. Ann Hum Genet 2000; 64(4): $433-442$.

12 Kauffmann F, Dizier MH, Pin I et al: Epidemiological study on the genetics and environment of asthma, bronchial hyperresponsiveness and atopy (EGEA)-Phenotype issues. Am J Respir Crit Care Med 1997; 156: S123-S129.

13 Kauffmann F, Dizier MH, Annesi-Maesano I et al: Etude épidémiologique des facteurs Génétiques et Environnementaux de l'Asthme, l'hyperréactivité bronchique et l'atopie (EGEA) Protocole et biais de sélection potentiels. Rev Epidémiol Santé Pub (in press)

14 Oryszczyn M P, Annesi-Maesano I, Charpin D, Paty E, Maccario J, Kauffmann F: Relationships of active and passive smoking to total IgE in adults of EGEA - (Epidemiological study on the genetics and Environment of Asthma, bronchial hyperresponsiveness and atopy). Am J Respir Crit Care Med 2000; 161: 1241 1246.

15 Anonymous. 1993. United Medical and dental Schools of Guy's and St Thomas's Hospitals, Department of Public Health Medicine. Protocol for the European Community respiratory Health Survey. ISBN 1869942019 London.

16 Risch N. Linkage strategies for genetically complex traits III. The effect of Marker polymorphism on analysis of affected relative pairs. Am J Hum Genet 1990; 46: 242 - 253.

17 Suarez BK: The affected sib pair IBD distribution for HLA-linked disease susceptibility genes. Tissue Antigens 1978; 12: 87-93.

18 Holmans P: Asymptotic properties of affected sib-pair linkage analysis. Am J Hum Genet 1993; 52: 362-374.

19 Self SG, Liang KY: Asymptotic properties of maximum likelihood estimators and likelihood ratio tests under non-standard conditions. J Am Stat Assoc 1987; 82: 605 - 610.

20 Morton NE: The detection and estimation of linkage between the genes for elliptocytosis and the Rh blood type. Am J Hum Genet 1956; 8: 80-96. 
21 Quesneville H, Dizier MH, Clerget-Darpoux F. Departure from the triangle constraints in discordant sib pairs: a test for genetic heterogeneity. Genet Epidemiol 1999; 17: 685 - 689.

22 Hizawa N, Freidhoff LR, Chiu YF et al: Genetic regulation of Dermatophagoides pteronyssinus-specific IgE responsiveness: A genome-wide multipoint linkage analysis in families recruited through 2 asthmatic sibs. J Allergy Clin Immunol 1998; 102: 436442.

23 Ober C, Tsalenko A, Willadsen S et al: Genome-wide screen for atopy susceptibility alleles in the Hutterites. Clin Exp Allergy 1999; 29: S11-S15.

24 Ober C, Tsalenko A, Parry R, Cox NJ: A second-generation genomewide screen for asthma-susceptibility alleles in a founder population. Am J Hum Genet 2000; 67: 1154-1162.

$25 \mathrm{Wjst} \mathrm{M}$. for the German asthma genetics group: Specific IgE-one gene fits all? Clin. Exp. Allergy 1999; 29: S5-S10.
26 Yokouchi Y, Nukaga Y, Shibasaki M et al: Significant evidence for linkage of mite-sensitive childhood to chromosome 5q31-q33 near the interleukin 12B locus by a genome-wide search in Japanese families. Genomics 2000; 66: 152-160.

$27 \mathrm{Xu}$ J, Postma DS, Howard TD et al: Major genes regulating total serum immunoglobulin E levels in families with asthma. Am J Hum Genet 2000; 67: 1163 - 1173.

28 Dahl M, Tyaer-Hansen A, Lange P, Nordestgaard BG: $\Delta$ F508 heterozygosity in cystic fibrosis and susceptibility to asthma. Lancet 1998; 351: 1911-1912.

29 Lazaro C, de Cid R, Sunyer J et al: Missense mutations in the cystic fibrosis gene in adult patients with asthma. Hum Mutat 1999; 14: 510-519.

30 De Cid R, Chomel JC, Lazaro C et al: CFTR and asthma in the French EGEA study. Eur J Hum Gen 2001; 9: 67-69. 